\title{
Wrist injuries detected on magnetic resonance imaging in athletes participating in the Rio de Janeiro 2016 Summer Olympic Games
}

\author{
Hamza Alizai ${ }^{1}$, Lars Engebretsen ${ }^{2,3,4}$, Mohamed Jarraya ${ }^{5,6}$, Frank W. Roemer ${ }^{5,7}$, Ali Guermazi ${ }^{5}$ \\ ${ }^{1}$ Department of Radiology, Scottish Rite for Children, Dallas, TX, USA; ${ }^{2}$ Medical and Scientific Department, International Olympic Committee, \\ Lausanne, Switzerland; ${ }^{3}$ Oslo Sports Trauma Research Center, Department of Sports Medicine, Norwegian School of Sport Sciences, Oslo, \\ Norway; ${ }^{4}$ Department of Orthopedic Surgery, Oslo University Hospital, University of Oslo, Norway; ${ }^{5}$ Quantitative Imaging Center, Department of \\ Radiology, Boston University School of Medicine, Boston, MA, USA; ${ }^{6}$ Department of Radiology, Mercy Catholic Medical Center, Darby, PA, USA; \\ ${ }^{7}$ Department of Radiology, University of Erlangen-Nuremberg, Erlangen, Germany
}

Correspondence to: Hamza Alizai. Department of Radiology, Scottish Rite for Children, 2222 Welborn St, Dallas, TX, 75219, USA. Email: hamzaalizai@gmail.com.

\begin{abstract}
Background: Traumatic and chronic overuse injuries of the wrist are common in athletes. The purpose of this study was to describe the frequency, anatomic distribution, and severity of MRI-detected wrist joint injuries amongst athletes who competed in the Rio de Janeiro 2016 Summer Olympics.

Methods: All sports injuries reported by the National Olympic Committee medical teams and the Organizing Committee medical staff during the 2016 Summer Olympics were analyzed. MRI was performed at the International Olympic Committee's polyclinic within the Olympic Village, using 3T and 1.5T scanners. The MRIs were interpreted by musculoskeletal radiologists with expertise in sports injuries. The distribution of wrist injuries by anatomic location and sports discipline and the severity of injuries were recorded.
\end{abstract}

Results: A total of 1,101 injuries were reported in the 11,274 athletes. Twenty-five athletes (72\% male, median-age $=27$ years $)$ had an MRI for wrist injuries. Fifty-six percent $(\mathrm{N}=14)$ of these athletes had triangular fibrocartilage pathology, $64 \%$ of which were chronic, while $36 \%$ were acute. There were scapholunate ligament injuries in $40 \%$ of the athletes. The extensor carpi ulnaris tendon was most commonly injured tendon. Fractures were seen in $32 \%$ of the athletes $(\mathrm{N}=8)$ and most commonly involved the scaphoid. Athletes participating in weightlifting $(\mathrm{N}=4,16 \%)$, tennis $(\mathrm{N}=3,12 \%)$ and gymnastics $(\mathrm{N}=3,12 \%)$ athletes were most commonly affected.

Conclusions: MRI-detected wrist injuries during the 2016 Summer Olympics most commonly affected the scapholunate ligament, extensor carpi ulnaris tendon and triangular fibrocartilage articular disc. The highest occurrence of wrist injuries was in weightlifting, tennis and gymnastics

Keywords: Wrist injuries, Olympic Games; Magnetic Resonance Imaging

Submitted Oct 06, 2020. Accepted for publication Apr 07, 2021.

doi: 10.21037/qims-20-1121

View this article at: http://dx.doi.org/10.21037/qims-20-1121

\section{Introduction}

Injuries involving the wrist and hand comprise up to $9 \%$ of all sports-related injuries (1). Acute traumatic wrist injuries are more common in contact sports (1), while chronic overuse injuries are generally seen in non-contact events such as the racquet sports (2). The epidemiology of wrist injuries is well understood. The data regarding wrist pathology detected by MRI during the Olympic Games has however not been published (3).

Effective injury prevention programs usually include injury surveillance as an important component. The International Olympic Committee (IOC) has therefore 
instituted injury surveillance systems to detect risk factors and mechanisms of injuries suffered during the Olympics with the overall goal of protecting the health of participating elite athletes $(3,4)$. Medical imaging can greatly aid this effort as it presents quantifiable data for identification of injury patterns in various sports. Moreover, it has been widely utilized during recent Olympic games and particularly during the 2016 Summer Olympic Games (4). Retrospective analysis of this available imaging data should allow development of targeted preventative measures, improvement in management algorithms and help to determine the best allocation of therapeutic resources.

Magnetic resonance imaging (MRI), which we report on in this paper, allows unparalleled evaluation of structures in the wrist and can complement clinical assessment in the diagnosis of injuries and their severity. In light of these considerations, this study describes the demographics, incidence, anatomic distribution, and severity of MRIdetected wrist injuries involving athletes during the 2016 Summer Olympic Games in Rio de Janeiro, Brazil.

\section{Methods}

This study was approved by the International Olympic Committee (IOC). This is a retrospective analysis of imaging data collected at the Rio 2016 Summer Olympic Games and retrieved from the Radiological Information System (RIS) and Picture Archiving and Communication System (PACS). During the 2016 summer Olympic games, medical and imaging services were provided from the opening of the Olympic Village on July $24^{\text {th }}$ through closing on August 24 2016.

Daily occurrence of injuries was reported on a standardized medical report form by all participating National Olympic Committee (NOC) medical teams. This information was retrieved for the study. Clinical information was also retrieved for athletes treated for injuries in the polyclinic and all other medical venues by the medical staff of the Organizing Committee of the Rio de Janeiro 2016.

\section{Confidentiality and ethical approval}

This study was approved by the local institutional review board and individual consent for this retrospective analysis was waived. We obtained approval from the IOC to use anonymized imaging and demographic data for publication.

\section{Data collection}

GE Healthcare provided all imaging equipment according to an agreement with the International Olympic Committee. MRI studies were performed in the Olympic village using 3T Discovery MR750w and 1.5T Optima 450MRw MRI scanners (all machines GE Healthcare, Brazil). A total of 869 radiological examinations including 607 MRIs were performed. Standard clinical MRI protocols were used consisting of 3-plane acquisition of fluid-sensitive images (including fat-suppressed T2-weighted and/or fatsuppressed proton density-weighted), and T1-weighted images in at least one plane. Information on wrist injuries detected on imaging was retrospectively collected from the original radiology reports generated in the RIS. Athlete demographics were collected in an anonymized fashion for those athletes diagnosed with wrist injuries on MRI. The data were stratified by gender, age, participating country, and sports discipline.

\section{Imaging interpretation}

Two musculoskeletal radiologists with expertise in sports imaging independently reviewed the presence, anatomic location, and severity of wrist pathology on MRI.

The triangular fibrocartilage complex (TFCC) was graded based on the Palmer classification (5), which categorizes lesions as being either traumatic (class I) or degenerative (class II). Further subdivisions localize injury within the TFCC. Class IA represents central perforation of the TFC articular disc (focal discontinuity near the radial insertion); class IB is ulnar avulsion of the TFC (avulsion/ tear of the ulnar foveal and/or styloid attachments); class IC is traumatic avulsion of the peripheral attachments of the TFCC (specifically the ulnolunate or ulnotriquetral ligaments) and class ID represents traumatic avulsion of the radial attachment of the TFC in the region of sigmoid notch. Class IIA represents degenerative wear of the TFCC; class IIB is degenerative wear with lunate and/or ulnar chondromalacia; class IIC is TFCC perforation with lunate and/or ulnar chondromalacia; class IID is IIC plus a lunotriquetral ligament perforation; class IIE is IID plus ulnocarpal arthritis.

An MRI based grading system for the scapholunate (SL) and lunotriquetral (LT) ligaments has not been established. We graded the SL ligament on a Likert-scale based on the European Wrist Arthroscopic Society (EWAS) classification (6) as follows: (I) a tear of the membranous component 
(discontinuity of the membranous component observed on axial and/or coronal images); (II) a partial thickness tear of the volar component (partial discontinuity of the ligament); (III) a partial thickness tear of the dorsal component (partial discontinuity of the ligament); (VI) a complete tear (full thickness discontinuity) of the dorsal component without widening of the SL interval; (V) a complete tear of all components of the ligament (complete discontinuity) with widened SL interval. The lunotriquetral ligament was graded using the same classification scheme.

The tendons of the wrist were graded with the goal of distinguishing chronic tendinosis from acute injury. The flexor tendons of the wrist include the flexor carpi ulnaris, flexor digitorum superficialis, flexor digitorum profundus, palmaris longus and flexor carpi radialis tendons. The extensor tendons are located in six extensor compartments; the first compartment contains the abductor pollicis longus and extensor pollicis brevis tendons, the second compartment includes the extensor carpi radialis longus and brevis tendons, the third compartment consists of the extensor pollicis longus tendon, the fourth compartment contains the extensor digitorum and extensor indices tendons, the fifth compartment has the extensor digitiminimi tendon and the sixth compartment contains the extensor carpi ulnaris tendon. Since an MRI-based grading system of the tendons with clinical, surgical, and outcome relevance has yet to be developed, we graded wrist tendons on a Likert-scale based on the routine evaluation of tendons in our clinical practice. The tendons were classified as 0 : normal tendon; 1 : mild tendinosis (increased intrasubstance fluid signal intensity and no thickening of tendon); 2 : moderate tendinosis (increased intrasubstance fluid signal intensity with mild thickening of the tendon); 3: severe tendinosis (increased intrasubstance fluid signal intensity with marked thickening of the tendon); 4: low-grade partial tear (partial discontinuity of the tendon involving less than 50\% diameter); 5: high-grade partial tear (partial discontinuity of the tendon involving greater than $50 \%$ thickness); 6: full thickness tear (complete discontinuity of the tendon).

Severity of osseous injury was graded based on the classification proposed by Nattive et al. for assessment of stress fractures in athletes (7). With this classification scheme, 0: normal bone; 1: mild marrow edema or periosteal edema; 2: moderate marrow or periosteal edema; 3: severe marrow or periosteal edema; 4: fracture line in addition to severe marrow or periosteal edema. The location of the injury was recorded.

Radiocarpal joint effusion was graded from 0 to 3 in terms of the estimated maximal distention of the synovial cavity: 0: normal; $1:<33 \%$ of maximum potential distention; 2: $33 \%$ : $66 \%$ of maximum potential distention; $3:>66 \%$ of maximum potential distention (8). The presence or absence of a ganglion cyst was graded as a binary variable; 0 : absent and 1: present.

Finally, the ulnar and median nerves were assessed and graded using a scheme based on the Seddon classification: 0: normal, 1: abnormal signal intensity, 2: abnormal morphology (focal enlargement and/or increased signal intensity) (9).

After grading, any difference in opinion was adjudicated through mutual consensus by the radiologists following discussion.

\section{Results}

A total of 11,274 athletes participated in the Olympic games and were included in the IOC's surveillance study, amongst whom there were 1,101 reported injuries. Only 25 athletes underwent MRI for wrist injuries $(72 \%$ male, median age: 27 years, range 15-34 years). They represented 16 countries and thirteen sports disciplines (Figure 1) including aquatics, athletics, boxing, canoe, cycling, gymnastics, handball, hockey, judo, rugby, shooting, tennis, and weightlifting. Wrist injuries were seen in athletes from Europe (three from France, two from Great Britain, two from Serbia, two from Ukraine, one each from Germany, Belarus, Albania, Slovakia and Poland), Africa (two from South Africa, one from Ghana), Asia (two from Mongolia, two from Kazakhstan, one from Russia, one from India) and New Zealand (two). The number of athletes from each discipline who underwent MRI for wrist injury is presented in Table 1.

\section{Anatomical location of wrist pathology}

Tendon pathology was most common in athletes who underwent MRI for wrist injuries. The extensor carpi ulnaris (ECU) tendon was the most commonly injured tendon, with ECU abnormality seen in the wrist of 19 athletes. No flexor tendon abnormalities were present. Fourteen athletes had TFCC pathology. There were scapholunate ligament abnormalities in $40 \%$ of the athletes while only $12 \%$ had lunotriquetral ligament abnormality. Fractures were seen in $32 \%$ of the athletes 


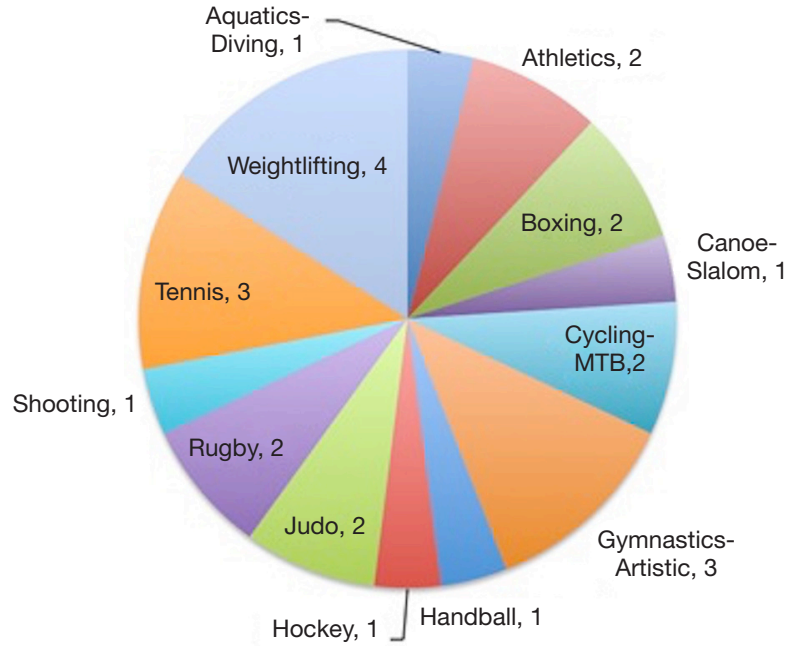

Figure 1 Wrist injuries by sport. Values represent the number of cases. MTB, mountain bike.

Table 1 Wrist injuries by sport

\begin{tabular}{lccc}
\hline Disciplines & $\begin{array}{c}\text { Number of } \\
\text { athletes }\end{array}$ & $\begin{array}{c}\text { Number of MRI } \\
\text { detected wrist } \\
\text { injuries }\end{array}$ & $\begin{array}{c}\text { Incidence } \\
\text { proportion } \\
(\%)\end{array}$ \\
\hline Aquatics: diving & 135 & 1 & 0.74 \\
Athletics & 2367 & 2 & 0.08 \\
Boxing & 286 & 2 & 0.70 \\
Canoe: slalom & 83 & 1 & 1.20 \\
Cycling: mountain bike & 80 & 2 & 2.50 \\
Gymnastics: artistic & 96 & 3 & 3.13 \\
Handball & 335 & 1 & 0.30 \\
Hockey & 384 & 1 & 0.26 \\
Judo & 390 & 2 & 0.51 \\
Rugby & 291 & 2 & 0.70 \\
Shooting & 390 & 1 & 0.26 \\
Tennis & 199 & 3 & 1.51 \\
Weightlifting & 256 & 4 & 1.56 \\
Total & 5,292 & 25 & 0.47 \\
\hline
\end{tabular}

$(\mathrm{N}=8)$ and most commonly involved the scaphoid. The majority of the athletes who underwent MRI for wrist injuries participated in weightlifting $(\mathrm{N}=4,16 \%)$, tennis $(\mathrm{N}=3,12 \%)$ and gymnastics $(\mathrm{N}=3,12 \%)$.

The following sections describe the distribution and severity of the detected pathology in more detail.
Table 2 Grades of ligament injuries

\begin{tabular}{lcc}
\hline Grade & $\mathrm{SL}, \mathrm{n}(\%)$ & $\mathrm{LT}, \mathrm{n}(\%)$ \\
\hline 1 & $6(60.0)$ & $1(33.3)$ \\
2 & $1(10.0)$ & $0(0.0)$ \\
3 & $2(20.0)$ & $2(66.6)$ \\
4 & $1(10.0)$ & $0(0.0)$ \\
Total cases & 10 & 3 \\
\hline
\end{tabular}

SL, scapholunate ligament; LT, lunotriquetral ligament.

\section{Ligament patbology}

Eleven athletes with wrist injuries who underwent MRI had an abnormality of either the scapholunate or the lunotriquetral ligament. Two athletes sustained injuries of both of these ligaments, while one had an isolated injury of the lunotriquetral ligament. Of the 10 athletes with SL ligament injuries, six had tears of the intramembranous component of the ligament (Grade 1), one athlete suffered partial tear of the volar component (Grade 2), two had partial tears of the dorsal component (Grade 3) and one had a complete tear of the SL ligament without scapholunate interval widening (Grade 4). Two athletes had partial tears of the dorsal lunotriquetral ligament and 1 had a tear of the intramembranous component of the LT ligament. Table 2 summarizes the distribution and severity of ligamentous injuries according to the adapted EWAS classification.

\section{Tendon pathology}

Only extensor tendon abnormalities were seen and none of the athletes suffered an injury to their flexor tendons. Nineteen athletes with wrist injuries who underwent MRI had an abnormality of at least one tendon. All nineteen of these athletes had an abnormality of the extensor carpi ulnaris tendon, two-thirds of these injuries characterized as low-grade partial tears, which involved less than $50 \%$ tendon thickness. High-grade partial tears of the ECU were observed in two athletes who participated in tennis and athletics respectively (Figure 2).

Injury to other tendons was less common, with only mild to moderate tendinosis seen in the other extensor compartments. After the ECU, the second extensor compartment demonstrated most abnormalities, with six athletes having mild tendinosis of their extensor carpi 

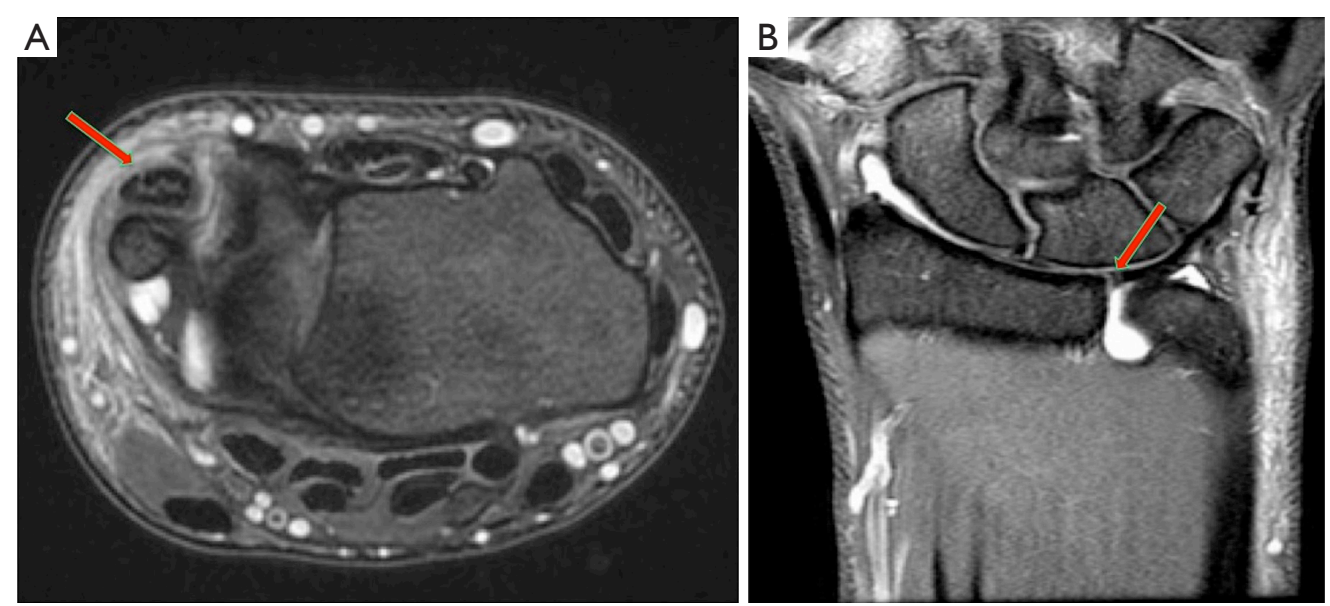

Figure $2 \mathrm{MRI}$ of this tennis athlete in his late twenties depicts abnormality of the extensor carpi ulnaris tendon and the triangular fibrocartilage articular disc. Axial proton density-weighted fat-suppressed MRI (A) depicts a longitudinal split tear of the extensor carpi ulnaris tendon. Note the extensive ulnar sided subcutaneous soft tissue edema. Also present were a tear of the extensor carpi ulnaris subsheath (not shown), central perforation of the triangular fibrocartilage articular disc and distal radioulnar joint effusion seen on the coronal proton density-weighted fat-suppressed MRI (B). The red arrows highlight the described pathology described in the figure captions.

Table 3 Grades of extensor tendon injuries by compartment

\begin{tabular}{|c|c|c|c|c|c|c|}
\hline Grade & First compartment & Second compartment & Third compartment & Fourth compartment & Fifth compartment & Sixth compartment \\
\hline 2 & $1(25 \%)$ & 0 & 0 & $1(33.3 \%)$ & 0 & $1(5.55 \%)$ \\
\hline 3 & 0 & 0 & 0 & 0 & 0 & 0 \\
\hline 4 & 0 & 0 & 0 & 0 & 0 & $12(66.6 \%)$ \\
\hline 6 & 0 & 0 & 0 & 0 & 0 & 0 \\
\hline Total cases & 4 & 6 & 3 & 3 & 0 & 18 \\
\hline
\end{tabular}

radialis brevis and longus tendons. Table 3 summarizes the distribution and severity of ligamentous pathology.

\section{Triangular fibrocartilage injuries}

Fifty-six percent $(\mathrm{N}=14)$ of the athletes had triangular fibrocartilage pathology, 64\% of which were chronic while $36 \%$ were acute injuries. The most common acute injury involved a central perforation of the triangular fibrocartilage articular disc, which was seen in four athletes. One athlete, who participated in the shooting discipline sustained a peripheral ulnar sided tear of the disc (Figure 3). Amongst the athletes with degenerative abnormalities of the TFCC, triangular fibrocartilage disc perforation with lunate and/ or ulnar chondromalacia was most common (Palmer grade IIC). Table 4 summarizes the prevalence of TFCC injuries by Palmer classification grade.

\section{Miscellaneous other injuries}

Eight athletes who had MRI for wrist injuries were found to have fractures. Three of these athletes sustained acute scaphoid waist fractures and one was found to have a scaphoid non-united fracture with avascular necrosis of the proximal pole. Figure 4 depicts one of these athletes with an acute nondisplaced scaphoid waist fracture. Other fractures included an intraarticular second metacarpal base fracture in a tennis athlete, a triquetral avulsion fracture in a rugby 


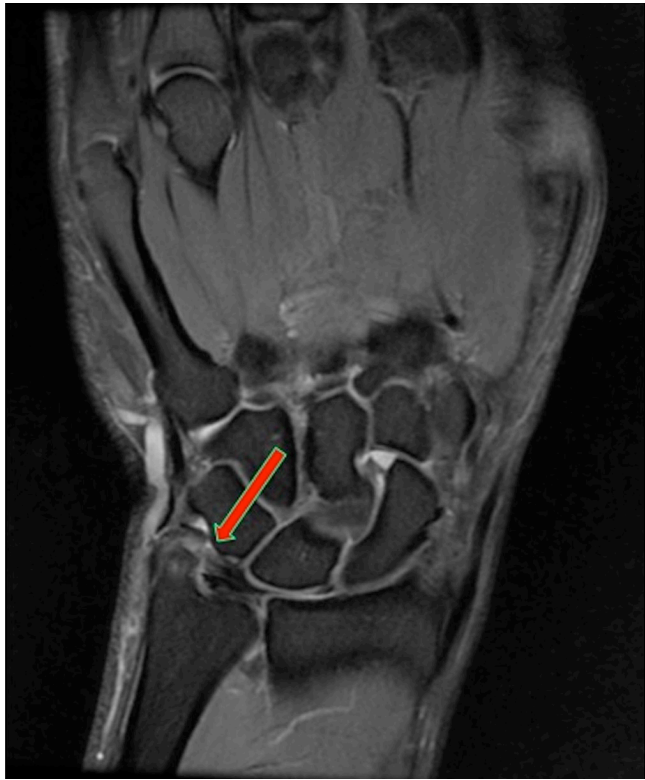

Figure 3 Coronal proton density-weighted fat-suppressed MRI of the wrist in a male athlete in his late twenties, who participated in shooting, depicts ulnar avulsion of the triangular fibrocartilage articular disc from its foveal and styloid attachments (Palmer IB). The red arrow highlight the described pathology described in the figure caption.

Table 4 Palmer classification of TFCC

\begin{tabular}{lc}
\hline Grade & Prevalence \\
\hline Class 1a & $4(28.5 \%)$ \\
Class 1b & $1(7.14 \%)$ \\
Class 1c & 0 \\
Class 1d & 0 \\
Class 2a & $3(21.4 \%)$ \\
Class 2b & $1(7.14 \%)$ \\
Class 2c & $5(35.7 \%)$ \\
Class 2d & 0 \\
Class 2e & 0 \\
Total cases & 14 \\
\hline
\end{tabular}

TFCC, triangular fibrocartilage complex.

player, a hamate hook fracture in a cyclist and a SalterHarris one fracture of the distal radius in a gymnast.

Nine out of the 25 athletes who had a wrist MRI $(36 \%)$ were found to have a ganglion cyst. These ganglion

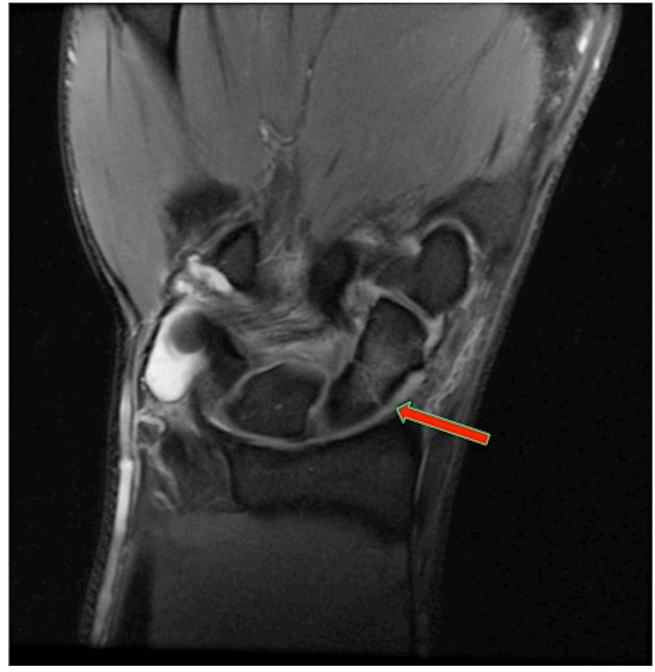

Figure 4 Coronal proton density-weighted fat-suppressed MRI of the wrist in a male athlete in his early twenties depicts a nondisplaced scaphoid waist fracture with associated bone marrow edema. Also, there is a joint effusion. The red arrows highlight the described pathology described in the figure captions.

cysts ranged from 5 to $25 \mathrm{~mm}$ in size. No morphologic abnormality of the median or ulnar was seen in any of the athletes. Six athletes (24\%) had small grade 1 radiocarpal joint effusions. None had any intraarticular loose bodies.

\section{Discussion}

Incorrect or delayed diagnosis of wrist injuries may lead to complications with functional sequela (10). MRI allows unsurpassed noninvasive evaluation of the wrist; it is $100 \%$ sensitive and specific for the diagnosis of occult fractures (11), 100\% sensitive and 90\% specific for TFCC tears (12), a sensitivity of $70-81 \%$ and specificity of up to $100 \%$ for scapholunate ligament tears (13). The sensitivity, specificity and accuracy of $3 \mathrm{~T}$ MRI is higher compared to $1.5 \mathrm{~T}$ MRI, particularly for TFCC injuries (14). This retrospective analysis included 25 athletes who underwent MRI for wrist injuries suffered during the Rio de Janeiro 2016 Summer Olympic Games. These 25 athletes represented thirteen sports disciplines and equated to about just about $0.5 \%$ of the 5,292 athletes in those disciplines. We suspect that the reason for an incidence rate lower than previously published is that only athletes who underwent MRI were included in this study and those who may have been diagnosed clinically but not imaged were not part of 
this retrospective study.

To date, data regarding wrist injuries detected using MRI during any of the previous summer Olympic Games has not been published. The athletes who most commonly underwent MRI for wrist injuries during the 2016 summer Olympic games included those participating in tennis, weightlifting and gymnastics. Overuse injuries are known to be prevalent in athletes participating in these sports, which require repetitive wrist loading. Salter-Harris 1 injury has been described in gymnasts and was found in one of the 3 gymnasts who underwent wrist MRI. In this case, a SalterHarris 1 injury was clinically suspected and periphyseal edema was noted on MRI. The scaphoid is the most commonly injured carpal bone, usually occurring as part of a hyperextension wrist injury in a pronated, radially deviated hand (15). Half of the athletes with fractures in this study had a scaphoid waist fracture, with one of these being an old non-united fracture with evidence of Stage 2 scapholunate advanced collapse. The other acute fractures included a hamate hook fracture, a $3^{\text {rd }}$ metacarpal base fracture, and a triquetral avulsion fracture. A 28-year-old athlete who participated in boxing showed signs of mild to moderate osteoarthritis at the second and third carpometacarpal joints.

Amongst the twenty-five athletes, the most common abnormality was that of the extensor carpi ulnaris tendon. ECU injuries are known to be common in racquet sports and are thought to be the sequela of repetitive microtrauma or sudden traumatic episode during wrist flexion, supination, and ulnar deviation (15). ECU pathology is commonly found in conjunction with TFCC abnormality, as the TFCC is continuous with the ECU subsheath. Of the 5 athletes with acute TFCC injuries in this study, 3 had concomitant abnormalities of the ECU (2 partial tears and 1 moderate tendinosis). Of the 9 athletes with chronic abnormalities of the TFCC, 7 had concurrent abnormalities of the ECU (6 with low-grade partial tears and 1 with mild tendinosis).

Limitations of this retrospective study may include selection bias. We assume that not all athletes who suffered wrist injuries underwent MRI during the Olympics. This is not therefore a study of prevalence of wrist injuries, but rather a description of the pathologies in athletes with wrist complaints who underwent MRI during the 2016 summer Olympics. Furthermore, the differentiation between acute and chronic injuries is based on the imaging appearance rather than a clinical history. Data regarding clinical management, recovery from the injuries and return to sports was not available.

\section{Conclusions}

This study included 25 athletes who had MRI for wrist injuries during the Rio de Janeiro 2016 Summer Olympic Games. ECU was the most common site of abnormality found on MRI, followed in order by TFCC and SL ligament. Fractures were found in a third of the athletes. Although a physical examination can direct an expert clinician towards the diagnosis, MRI provides an unparalleled anatomical evaluation of the wrist and can help accurately determine the structures injured and the severity of these injuries. With this information, epidemiological studies can help sports clinicians to predict injuries and optimize the diagnosis and management of wrist injuries during high-level athletic events such as the Olympic Games.

\section{Acknowledgments}

This manuscript has not been previously published or submitted for publication elsewhere except as a brief abstract in the proceedings of a scientific meeting or symposium. The abstract of our manuscript was submitted to the 2019 European Congress of Radiology for scientific exchange and was accepted as a poster presentation. All accepted original science presentations have been published in the book of abstracts by the European Society of Radiology.

Funding: None.

\section{Footnote}

Conflicts of Interest: All authors have completed the ICMJE uniform disclosure form (available at http://dx.doi. org/10.21037/qims-20-1121). Dr. AL serves as an unpaid editorial board member of Quantitative Imaging in Medicine and Surgery, he reports per-sonal fees from BICL, LLC, personal fees from Pfizer, AstraZeneca, MerckSerono, Tis-sueGene, OrthoTrophix, GE, Sanofi, outside the submitted work. Dr. FWR reports other from Boston Imaging Core Lab. (BICL), LLC., outside the submitted work. The other authors have no conflicts of interest to declare.

Ethical Statement: Our retrospective study was reviewed by the Medical Research Ethics Committee of the SouthEastern Norway Regional Health Authority (2011/388) and was exempted from Ethics Committee approval. Informed consent was waived since all data in our epidemiological study were anonymized and unidentifiable. We obtained 
approval from the Internal Olympic Committee (IOC) to use anonymized imaging and demo-graphic data for publication.

Open Access Statement: This is an Open Access article distributed in accordance with the Creative Commons Attribution-NonCommercial-NoDerivs 4.0 International License (CC BY-NC-ND 4.0), which permits the noncommercial replication and distribution of the article with the strict proviso that no changes or edits are made and the original work is properly cited (including links to both the formal publication through the relevant DOI and the license). See: https://creativecommons.org/licenses/by-ncnd/4.0/.

\section{References}

1. Rettig AC. Athletic injuries of the wrist and hand. Part I: traumatic injuries of the wrist. Am J Sports Med 2003;31:1038-48.

2. Rettig AC. Athletic injuries of the wrist and hand: part II: overuse injuries of the wrist and traumatic injuries to the hand. Am J Sports Med 2004;32:262-73.

3. Engebretsen L, Soligard T, Steffen K, Alonso JM, Aubry M, Budgett R, Dvorak J, Jegathesan M, Meeuwisse WH, Mountjoy M, Palmer-Green D, Vanhegan I, Renström PA. Sports injuries and illnesses during the London Summer Olympic Games 2012. Br J Sports Med 2013;47:407-14.

4. Guermazi A, Hayashi D, Jarraya M, Crema MD, Bahr R, Roemer FW, Grangeiro J, Budgett RG, Soligard T, Domingues R, Skaf A, Engebretsen L. Sports Injuries at the Rio de Janeiro 2016 Summer Olympics: Use of Diagnostic Imaging Services. Radiology 2018;287:922-32.

5. Oneson SR, Scales LM, Timins ME, Erickson SJ, Chamoy L. MR imaging interpretation of the Palmer classification of triangular fibrocartilage complex lesions. Radiographics 1996;16:97-106.

Cite this article as: Alizai $\mathrm{H}$, Engebretsen L, Jarraya M, Roemer FW, Guermazi A. Wrist injuries detected on magnetic resonance imaging in athletes participating in the Rio de Janeiro 2016 Summer Olympic Games. Quant Imaging Med Surg 2021;11(7):3244-3251. doi: 10.21037/qims-20-1121
6. Messina JC, Van Overstraeten L, Luchetti R, Fairplay T, Mathoulin CL. The EWAS Classification of Scapholunate Tears: An Anatomical Arthroscopic Study. J Wrist Surg 2013;2:105-9.

7. Nattiv A, Kennedy G, Barrack MT, Abdelkerim A, Goolsby MA, Arends JC, Seeger LL. Correlation of MRI grading of bone stress injuries with clinical risk factors and return to play: a 5-year prospective study in collegiate track and field athletes. Am J Sports Med 2013;41:1930-41.

8. Peterfy CG, Guermazi A, Zaim S, Tirman PF, Miaux Y, White D, Kothari M, Lu Y, Fye K, Zhao S, Genant HK. Whole-Organ Magnetic Resonance Imaging Score (WORMS) of the knee in osteoarthritis. Osteoarthritis Cartilage 2004;12:177-90.

9. Mitchell CH, Brushart TM, Ahlawat S, Belzberg AJ, Carrino JA, Fayad LM. MRI of sports-related peripheral nerve injuries. AJR Am J Roentgenol 2014;203:1075-84.

10. Pulos N, Kakar S. Hand and Wrist Injuries: Common Problems and Solutions. Clin Sports Med 2018;37:217-43.

11. Gaebler C, Kukla C, Breitenseher M, Trattnig S, Mittlboeck M, Vécsei V. Magnetic resonance imaging of occult scaphoid fractures. J Trauma 1996;41:73-6.

12. Potter HG, Asnis-Ernberg L, Weiland AJ, Hotchkiss RN, Peterson MG, McCormack RR. The utility of highresolution magnetic resonance imaging in the evaluation of the triangular fibrocartilage complex of the wrist. J Bone Joint Surg Am 1997;79:1675-84.

13. Spaans AJ, Minnen P, Prins HJ, Korteweg MA, Schuurman AH. The value of 3.0-tesla MRI in diagnosing scapholunate ligament injury. J Wrist Surg 2013;2:69-72.

14. Anderson ML, Skinner JA, Felmlee JP, Berger RA, Amrami KK. Diagnostic comparison of 1.5 Tesla and 3.0 Tesla preoperative MRI of the wrist in patients with ulnarsided wrist pain. J Hand Surg Am 2008;33:1153-9.

15. Avery DM, Rodner CM, Edgar CM. Sports-related wrist and hand injuries: a review. J Orthop Surg Res 2016;11:99. 